\title{
Formation of nerve impulse and its properties of transport with the terahertz wave along the nerve fiber in the life
} systems

\author{
Pang-Xiao Feng*, zeng-hong juan, Jiang-Ying Rui and Ni-Yin Jia \\ Institute of life science and technology, University of Electronic Science and Technology of China, China
}

We gave first the formation and properties of the action electropotential on the nerve-cell membranes, which are directly related to the non-uniformity of distribution of sodium ions and potassium ions between the inner and exterior of cell-membranes as well as their properties of movements under the affection of an electroinformation. However, the action electro-potential in essence is only a static impulse, it cannot propagate along the nerve organizations. But, if the nerve organizations are acted by the bio-energy, which could lead to the periodic variation of these sodium ions and potassium ions in the inner and exterior of the never cell membranes can be varied periodically under the action of bio-energy by virtue of the works of sodium pump and potassium pump on the surface of membrane of cells, then the nerve impulse can propagate along the nerve fiber cell membranes. Our investigations verify that the bio-energy released from the hydrolyses reaction of adenosine phosphate (ATP) molecules in the cells can play the role, it released the bio-energy of $0.42 \mathrm{eV}$ can be transported by the protein molecules to these sodium pump and potassium pump to work, thus the propagation of the nerve impulse can be carried out automatically in living systems. This is just the mechanism of propagation of the nerve impulse along the nerve cell membrane. This means that the energy released from the hydrolyses reaction of ATP molecules controlled the propagation of the nerve impulse. Because ATP molecules are often attached on the protein molecules, where the energy is transported along the protein molecules from the position of generation of hydrolyses reaction to the position used the energy in virtue of transport of the soliton formed by the excitons through the mechanism of self-trapping, where the exciton is a quantum produced by the $\mathrm{C}=0$ stretching (or amide-I) vibrations. We studied and obtained the properties of transport of bio-energy, which is carried by Pang's soliton, along $\alpha$-helical protein molecules and found further the lifetimes of Pang'soliton, which is between $0.53 \times 10$ ${ }^{10} \mathrm{~S} 0.65 \times 10^{-10} \mathrm{~S}$ at physiological temperature $\mathrm{T}=300 \mathrm{~K}$. In this lifetime Pang's soliton can travel over several hundreds of amino acid residues. This implies that Pang 's theory is a relevant and correct theory of bioenergy transport, then Pang's soliton is a real carrier of bio-energy transport in protein molecules. If the bio-energy was transported into the nerve membrane to drive the works of sodium pump and potassium pump, then the transfer of nerve impulse along the nerve membranes can be carried out. So, we can say that there is not the transport of nerve impulse without the works of sodium pump and potassium pump, or the bio-energy. This means that the nerve impulse can be transported along the nerve membrane, only if the bio-energy was provided and was absorbed really by the sodium pump and potassium pump. In order to form a stable propagation of nerve impulse, then its times forming must be shorter than the lifetime of Pang's soliton or its experimental values, or else, the nerve impulse is not stable and is useless. Thus, we can affirm that the nerve impulse is a terahertz wave. Thus, we affirmed and verified that the nerve impulse can be transport along the nerve systems in the terahertz wave, instead the millimeter wave. We determinate and discuss further its features. This is first time to determinate the terahertz features of transport of the nerve impulse along the nerve fibers in life systems, which will promote great the development of the nerve science [1].

\section{References}

1. Pang-Xiao feng (2018) The terahertz features of transport of nerve impulse along the nerve system driven by the bio-energy in life systems. Bionature 38: 146-213.
Copyright: (C)2018 Pang-Xiao F. This is an open-access article distributed under the terms of the Creative Commons Attribution License, which permits unrestricted use, distribution, and reproduction in any medium, provided the original author and source are credited.
*Correspondence to: Pang-Xiao Feng, Institute of life science and technology, University of Electronic Science and Technology of China, China, E-mail: pangxf2006@aliyun.com

Received: September 01, 2018; Accepted: September 14, 2018; Published: September 17, 2018 